\title{
Soy use and vasomotor symptoms: Soy Estrogen Alternative follow-up study
}

This article was published in the following Dove Press journal:

International Journal of Women's Health

I November 2010

Number of times this article has been viewed

\section{Mara Z Vitolins' \\ L Douglas Case' \\ Timothy M Morgan' \\ Margaret A Miller ${ }^{2}$ \\ Gregory L Burke'}

'Division of Public Health Sciences, Wake Forest University Health Sciences, Winston-Salem, North Carolina, ${ }^{2}$ National Center for Toxicology Research, Food and Drug Administration Rockville, Maryland, USA
Correspondence: Mara Z Vitolins Division of Public Health Sciences, Wake Forest University School of Medicine, Medical Center Boulevard, Winston-Salem, NC 27I57-000I, USA $\mathrm{Tel}+\mathrm{I} 3367162886$

Fax +13367134300

Email mvitolin@wfubmc.edu
Purpose: To evaluate vasomotor symptoms and soy and hormone therapy use in women who had previously participated in the Soy Estrogen Alternative (SEA) study, a trial conducted to compare the effects of soy protein supplements containing differing levels of isoflavones on menopausal symptoms, chronic disease risk factors, and health-related quality of life in perimenopausal and postmenopausal women.

Participants and methods: Two years after the SEA study ended participants were recontacted to complete questionnaires to quantify their health status, medications, menopausal symptoms, and their use of hormone therapy and soy-based foods and supplements. Participants were also asked to record vasomotor symptoms for seven days.

Results: Surveys were collected from 182 of the 241 participants who had been enrolled in the SEA study ( $76 \%$ response rate). Women were $55 \pm 2.8$ years of age, well educated $(80 \%$ more than high school), and 93\% reported good to excellent health. All but six reported experiencing at least one menopausal symptom, and 56\% reported one or more hot flashes on one or more days. Eighty-one women (45\%) continued to use soy for menopausal symptom relief, and $58(32 \%)$ were using hormone therapy. Women taking hormone therapy were experiencing fewer and less severe hot flashes than those who were not taking hormone therapy $(P<0.001)$; hot flash frequency and severity did not differ significantly between those who did and did not use soy, after controlling for hormone therapy use.

Conclusion: Most participants reported they were still experiencing menopausal symptoms. Additionally, half of the most symptomatic women (not taking hormone therapy) were still consuming soy products for vasomotor symptoms.

Keywords: menopause, vasomotor symptoms, soy consumption, survey

\section{Introduction}

It has been reported that $75 \%$ of menopausal women experience vasomotor symptoms and that these symptoms can markedly impact quality of life. ${ }^{1,2}$ Complementary and alternative medicine use is high in women seeking relief from the vasomotor symptoms of menopause. Soy consumption to treat menopausal symptoms has been studied as a result of cross-cultural comparisons showing that women who live in countries where substantial amounts of soy are consumed (ie, Japan and the Pacific Rim) not only have lower rates of coronary heart disease, fractures, and uterine or breast cancer, but also report fewer menopausal symptoms than women who live in countries where people consume very little soy in their diet (ie, the US and Western Europe)..$^{3-5}$

The Soy Estrogen Alternative (SEA) study was conducted to compare the effects of soy protein supplements containing differing levels of isoflavones (plant phytoestrogens) on menopausal symptoms, chronic disease risk factors, and health-related 
quality of life in perimenopausal and postmenopausal women. The women who participated in the SEA study were seeking vasomotor symptom relief and, although eligible for hormone therapy, did not want to use it.

The purpose of this paper is to report the results of a follow-up survey that was sent to the SEA participants two or more years following their closeout visit for the SEA study. We describe their self-reported vasomotor and other menopausal symptoms in relationship to hormone therapy use and soy consumption.

\section{Materials and methods Soy Estrogen Alternative study}

The SEA study was a double-blind randomized study to compare the effects of dietary supplements containing differing levels of isoflavones on menopausal symptoms, chronic disease risk, and health-related quality of life in perimenopausal and menopausal women. To be eligible for the SEA study, women had to be $45-55$ years old, have had no more than one menstrual period three months prior to enrollment, be healthy, have at least one vasomotor symptom in a 24 -hour period, and not be using traditional hormone replacement therapy or willing to discontinue use (three-month washout). A total of 241 participants were enrolled. The SEA study investigators reported that there was a significant reduction in the number and severity of symptoms during the 24-month follow-up period in all SEA treatment groups $(P<0.001)$. However, there were no significant differences at 24 months between the three groups $(P=0.10)$. A complete report of the study methodology and results are published elsewhere. ${ }^{6}$

\section{Follow-up survey}

Two or more years after their final (24-month) SEA study visit, a survey questionnaire was sent to all 241 participants. The survey contained questions about current health and reproductive status, prescription medications, menopausal symptoms, and use of soy products. Participants were asked about the use of soy foods (yes/no), soy/isoflavone pills (yes/no), and herbal products (yes/no) for the treatment of hot flashes. Careful attention was paid to limit participant burden, and the questionnaire was pretested prior to sending it to the study participants.

\section{Recontacting participants}

Participants were sent a cover letter explaining the study, an informed consent form, and the self-administered survey questionnaire. The women were asked to return the completed survey questionnaire and signed consent in the stamped, addressed envelope enclosed. Women were sent a reminder postcard two weeks after the survey questionnaires were sent out, and, at four weeks, nonresponders were contacted by telephone. The research was in compliance with the Helsinki Declaration, and the Wake Forest University School of Medicine Institutional Review Board approved the study and all study materials.

\section{Statistical analysis}

Participants were asked to record hot flash symptoms over a seven-day period. The number and severity $(0=$ none, $1=$ mild, 2 = moderate, and $3=$ severe) of hot flashes were recorded each day. Note that each hot flash was not given a severity rating, but rather the average severity rating for all the daily hot flashes was reported. The total number of hot flashes was simply the sum of the daily hot flashes over the seven-day period. Mean severity was calculated as the mean of the daily severity ratings. A daily hot flash score was calculated as the number of hot flashes times the average daily severity, and the mean hot flash score was calculated as the mean of the daily hot flash scores across the seven days. Participants had previously completed a diary for the closeout visit of the original study, and the vasomotor symptom data from that previous visit (calculated as described above) were added to the follow-up data file so that we could assess changes over time in vasomotor symptoms.

Means \pm standard deviation (SD) and percentages were generated to describe the characteristics of the SEA follow-up participants. For race, black and other were combined, incomes were dichotomized above and below $\$ 45,000$, education was dichotomized as equal to or below high school or greater than high school, body mass index (BMI) was categorized into four groups based on the Centers for Disease Control criteria, underweight (BMI $\left.<18.5 \mathrm{~kg} / \mathrm{m}^{2}\right)$, normal weight (BMI 18.5-25), overweight (BMI 25-30), obese class $\mathrm{I}$ ( $\mathrm{BMI} \geq 30$ ). The underweight and normal weight categories were combined for the analyses. Chi-squared tests and Kruskal-Wallis tests were used to assess the univariate associations between participant characteristics and soy/ hormonal therapy use. Logistic regression models were used to determine which characteristics (of those shown in Table 1) were jointly predictive of soy and hormonal therapy use and to determine which factors were associated with continued hot flashes. Linear regression on ranks was used to determine which factors were associated with the number of hot flashes and the hot flash score. Pearson correlation coefficients were used to assess the association between vasomotor symptoms of the SEA participants at closeout and vasomotor symptoms 
Table I Factors associated with soy and hormone therapy use among SEA follow-up participants*

\begin{tabular}{|c|c|c|c|c|c|}
\hline Characteristic & $\frac{\text { Neither soy nor } \mathrm{HT}}{\mathrm{n}(\%)}$ & $\frac{\text { Soy only }}{\mathrm{n}(\%)}$ & $\frac{\text { HT only }}{\text { n (\%) }}$ & $\frac{\text { Soy and } \mathrm{HT}}{\mathrm{n}(\%)}$ & $P$ value \\
\hline Total & $62(100)$ & $62(100)$ & $39(100)$ & $19(100)$ & \\
\hline Age (years) & & & & & 0.555 \\
\hline$<55$ & $33(53)$ & $32(52)$ & $17(44)$ & $12(63)$ & \\
\hline$\geq 55$ & $29(47)$ & $30(48)$ & $22(56)$ & $7(37)$ & \\
\hline Race & & & & & 0.655 \\
\hline White & $56(92)$ & $54(87)$ & $36(92)$ & $16(84)$ & \\
\hline Other & $5(8)$ & $8(13)$ & $3(8)$ & $3(16)$ & \\
\hline Income & & & & & 0.063 \\
\hline$<\$ 45,000$ & $30(52)$ & $23(38)$ & $10(26)$ & $6(32)$ & \\
\hline$\geq \$ 45,000$ & $28(48)$ & $37(62)$ & $29(74)$ & $13(68)$ & \\
\hline Education & & & & & 0.555 \\
\hline$\leq$ High school & $14(23)$ & $12(19)$ & $5(13)$ & $5(26)$ & \\
\hline$>$ High school & 47 (77) & $50(8 I)$ & $34(87)$ & $14(74)$ & \\
\hline BMI & & & & & 0.057 \\
\hline Underweight-normal & $24(39)$ & $24(39)$ & $21(54)$ & $13(68)$ & \\
\hline Overweight & $14(23)$ & $21(34)$ & $9(23)$ & $5(26)$ & \\
\hline Obese & $24(39)$ & $17(27)$ & $9(23)$ & I (5) & \\
\hline General health & & & & & 0.553 \\
\hline Excellent & $18(29)$ & $21(34)$ & $16(4 I)$ & $8(42)$ & \\
\hline Good & $39(63)$ & $35(56)$ & $22(56)$ & II (58) & \\
\hline Fair-poor & $5(8)$ & $6(10)$ & I (3) & $0(0)$ & \\
\hline Hysterectomy/oophorectomy & & & & & 0.646 \\
\hline No & $44(71)$ & $42(68)$ & $24(62)$ & II (58) & \\
\hline Yes & $18(29)$ & $20(32)$ & $15(38)$ & $8(42)$ & \\
\hline Medical conditions & & & & & 0.719 \\
\hline 0 & $31(50)$ & $31(50)$ & $16(41)$ & II (58) & \\
\hline 1 & $16(26)$ & $13(21)$ & $14(36)$ & $4(2 I)$ & \\
\hline $2+$ & $15(24)$ & $18(29)$ & $9(23)$ & $4(21)$ & \\
\hline Prescription medications & & & & & $<0.001$ \\
\hline 0 & $30(48)$ & $24(39)$ & $3(8)$ & I (5) & \\
\hline 1 & $13(2 \mid)$ & $17(27)$ & II (28) & $8(42)$ & \\
\hline 2 & $8(13)$ & $10(16)$ & $13(33)$ & $3(16)$ & \\
\hline $3+$ & $11(18)$ & $11(18)$ & $12(31)$ & $7(37)$ & \\
\hline Previous days with hot flashes/week** & & & & & 0.240 \\
\hline 0 & $9(24)$ & $12(26)$ & II (4I) & $3(23)$ & \\
\hline $1-6$ & $7(18)$ & $17(37)$ & $8(30)$ & $4(31)$ & \\
\hline 7 & $22(58)$ & $17(37)$ & $8(30)$ & $6(46)$ & \\
\hline Previous hot flashes/week ${ }^{* * *}$ & $17.8(14.8)$ & $17.8(26.8)$ & $14.3(18.4)$ & $15.8(18.7)$ & 0.555 \\
\hline Previous hot flash severity*** & $\mathrm{I} .06(0.8 \mathrm{I})$ & $0.88(0.8 \mathrm{I})$ & $0.74(0.84)$ & $0.93(0.75)$ & 0.348 \\
\hline Previous hot flash score ${ }^{* * *}$ & $4.17(4.16)$ & $4.96(10.5)$ & $3.85(5.85)$ & $3.43(4.18)$ & 0.567 \\
\hline
\end{tabular}

at follow-up. Paired $t$-tests were used to assess the change in these symptoms.

\section{Results}

One hundred and eighty-two women provided survey data for this study, representing a response rate of $76 \%$. Thirty-three survey packets were returned unopened, and all attempts to locate these women were unsuccessful. Twenty-five women declined the offer to participate and one woman was deceased.
Demographic characteristics for the 182 participants in this follow-up study are summarized by soy/hormonal therapy use in Table 1. Overall, 81 (45\%) of the 182 follow-up participants were using soy, including 58 for relief of hot flashes, two for relief of other menopausal symptoms, and 21 for both. Mean age was 55 years and most women were white and had more than a high school education. Ninety-three percent rated their health as good or excellent; with almost half of the women reporting that they had had no medical conditions in the past two years, and $68 \%$ responded that 
they were taking one or more prescribed medications. These characteristics were similar for women who did and did not use soy/hormonal therapy.

Forty-five percent of the women were using soy and $32 \%$ were taking hormonal therapy. Those who were not using hormonal therapy were more likely to be using soy than those who were using hormonal therapy (50\% versus $33 \%, P=0.03$ ). Hormonal therapy use was the only factor significantly associated with soy use in the multivariable logistic model. Soy use $(P=0.013)$, number of prescriptions $(P=0.003)$, and BMI $(P=0.037)$ were all significantly associated with hormonal therapy use in the logistic model. Women who were not taking soy, those with more prescriptions, and those with lower BMIs were more likely to use hormonal therapy. One hundred and twenty-four of the 182 women in the follow-up study had previously completed the original SEA study closeout visit. Seventy-two percent of those women were experiencing vasomotor symptoms at closeout, with a mean (SD) of 16.8 (20.9) symptoms per week. Those with vasomotor symptoms at the closeout visit were no more likely to be using soy or hormonal therapy at the follow-up visit than those who did not have vasomotor symptoms, unadjusted or adjusted for other factors.

Vasomotor symptoms recorded in the follow-up diaries are summarized in Table 2. Eighty women (44\%) did not experience hot flashes during the week they completed the diary, 55 (30\%) experienced at least one hot flash every day of the week, while the remaining 47 women (26\%) experienced hot flashes on 1-6 days. Thirty-three women (18\%) reported 1-7 hot flashes during the week, and 29 (16\%) reported 29 or more hot flashes. One participant experienced 164 hot flashes for the week. Women with lower incomes $(P=0.040)$ and those who did not use hormonal therapy $(P<0.001)$ were more likely to report having hot flashes.

The mean number of hot flashes was 12.0 (19.7) and was significantly lower for those using hormonal therapy (2.9 versus $16.3, P<0.001)$. No hot flashes were reported on $57 \%$ of the days for which diaries were kept, $1-5$ were reported for $33 \%$ of the days, $6-9$ for $8 \%$, and $\geq 10$ for $2 \%$ of the days. The daily hot flashes were typically mild to moderate. Only $12 \%$ of the days were recorded as severe. The mean hot flash score was 3.4 (7.3) and was significantly lower for those using hormonal therapy $(0.77$ versus 4.59 , $P<0.001)$.

Women who were experiencing hot flashes at the closeout visit of the original SEA study were more likely to have experienced them during the follow-up survey; 14 (40\%) of 35 women who reported no hot flashes at closeout reported them at follow-up compared with $57(64 \%)$ of 89 women who reported hot flashes at closeout $(P=0.01)$. Amongst the 124 women with vasomotor symptom data at closeout and follow-up, the number of weekly hot flashes dropped by a mean of $3.9(P=0.03)$ and the hot flash score decreased by 0.7 points $(P=0.17)$. The number of hot flashes at closeout was significantly correlated with the number at follow-up ( $\mathrm{r}=0.58, P<0.001$ ), as were the hot flash scores at closeout and follow-up ( $\mathrm{r}=0.71, P<0.001)$. In the multivariable linear models, only hormonal therapy and number of prior hot flashes were significantly associated with number of hot flashes or the hot flash score.

The number of menopausal and physical symptoms experienced by participants during the four weeks prior to

Table 2 Hot flashes and menopausal symptoms experienced by SEA follow-up participants over seven consecutive days by soy/HT use

\begin{tabular}{|c|c|c|c|c|c|}
\hline \multirow[t]{2}{*}{ Characteristic } & \multirow{2}{*}{$\begin{array}{l}\text { Neither soy nor HT } \\
\mathrm{n}(\%)\end{array}$} & \multirow{2}{*}{$\frac{\text { Soy only }}{\mathrm{n}(\%)}$} & \multirow{2}{*}{$\frac{\text { HT only }}{\text { n (\%) }}$} & \multirow{2}{*}{$\frac{\text { Soy and } \mathbf{H T}}{\mathrm{n}(\%)}$} & \multirow[t]{2}{*}{$P$ value } \\
\hline & & & & & \\
\hline Total & $62(100)$ & $62(100)$ & $39(100)$ & $19(100)$ & \\
\hline Days with hot flashes (n) & & & & & $<0.001$ \\
\hline 0 & $24(39)$ & $16(26)$ & $30(77)$ & $10(53)$ & \\
\hline $1-6$ & $13(2 \mid)$ & $21(34)$ & $5(13)$ & $8(42)$ & \\
\hline 7 & $25(40)$ & $25(40)$ & $4(10)$ & I (5) & \\
\hline Hot flashes (n) & $14.2(17.6)$ & $18.4(25.9)$ & $2.9(8.0)$ & $2.9(4.8)$ & $<0.001$ \\
\hline Hot flash severity (n) & $0.84(0.89)$ & I.0I $(0.90)$ & $0.24(0.60)$ & $0.35(0.56)$ & $<0.001$ \\
\hline Hot flash score (n) & $3.73(5.64)$ & $5.46(10.6)$ & $0.82(2.40)$ & $0.68(1.31)$ & $<0.00$ I \\
\hline Number of menopausal symptoms & & & & & 0.006 \\
\hline $0-5$ & $16(26)$ & $10(16)$ & $5(13)$ & $8(42)$ & \\
\hline $6-10$ & $10(16)$ & II (I8) & $18(46)$ & $3(16)$ & \\
\hline $1 \mathrm{I}-20$ & $22(35)$ & $27(44)$ & $13(33)$ & $6(32)$ & \\
\hline $21+$ & $14(23)$ & $14(23)$ & $3(8)$ & $2(11)$ & \\
\hline Menopausal symptoms (n) & I $3.0(8.3)$ & I4.I (7.4) & $10.6(6.1)$ & $9.7(7.9)$ & 0.043 \\
\hline
\end{tabular}

Abbreviations: SEA, Soy and Estrogen Alternative; HT, hormone therapy; SD, standard deviation. 
the follow-up survey was also evaluated. All but six women experienced at least one symptom, and the median number of symptoms experienced was 12. Joint pain and stiffness, general aches and pains, fatigue, bloating, and hot flashes were the most common symptoms, each occurring in more than $60 \%$ of the participants.

Women also reported their use of herbal supplements for menopausal symptoms. Thirty-five women (19\%) reported that they used herbal products for hot flashes. The most common herbal product taken for hot flashes was black cohosh (19 women). Twenty-eight women (15\%) reported using herbal products for other symptoms of menopause. The most common herbal products used for other symptoms were black cohosh and ginkgo biloba, both of which were taken by 10 women.

\section{Discussion}

An estimated two million American women are transitioning through menopause each year and are actively seeking therapies to treat their menopausal symptoms. ${ }^{7}$ This followup study was conducted to evaluate women who, when hormonal therapy was widely prescribed for vasomotor symptom management, opted to seek an alternative treatment for their menopausal symptoms by participating in the SEA study. Querying these participants a few years after the completion of the SEA study allowed for a longer-term evaluation of their menopausal symptoms and soy product consumption.

Women reporting having at least one vasomotor symptom over a 24-hour period met the eligibility criteria to participate in the SEA study. Of the 182 participants surveyed, more than half were still experiencing hot flashes, with 30\% having at least one every day of the week and $26 \%$ experiencing hot flashes on 1-6 days. Fifty-eight women were using hormonal therapy, and these women were experiencing fewer and less severe hot flashes. While hormonal therapy is effective in treating hot flashes, it is less effective in treating the other menopausal symptoms reported, including joint pain and stiffness, general aches and pains, fatigue, and bloating, and hormonal therapy has known deleterious health effects. This emphasizes the critical need for more research to identify and test longer-term treatments for menopausal symptoms.

An interesting finding was that half the women who were not taking HT and were still experiencing the most hot flashes, and those not taking hormonal therapy were using soy. There was no significant difference in hot flash frequency or severity between those who were and were not taking soy. In the original SEA study, each group had a significant decline in hot flash frequency and severity, even though the declines did not differ significantly by isoflavone dose. Although the isoflavones were not found to be responsible for reducing vasomotor symptoms in the SEA study, other components of soy may be responsible for the reduction of vasomotor symptoms. This seems plausible because $43 \%$ of the women reported that they continued to consume soy to treat their hot flashes (perhaps fearing that their symptoms might worsen if they stopped taking soy).

This study has some limitations that should be acknowledged. The data were collected from women who had participated in a soy intervention study and therefore were likely to be high utilizers of complementary and alternative therapies, and may be different from other menopausal women. However, with the highly publicized findings regarding hormonal therapy from the Women's Health Initiative Trial, more women experiencing symptoms of menopause are investigating alternative therapies with "lower" risk profiles to treat their hot flashes. ${ }^{8}$ Additionally, the literature supports the contention that women are using complementary and alternative medicine products for menopausal symptom management. Another limitation is that the data were selfreported.

\section{Conclusion}

This study adds to the current literature by evaluating vasomotor and other menopausal symptoms and soy/hormonal therapy use in a group of women who participated in a clinical trial testing an alternative therapy for vasomotor symptom management. We report that most of the women continued to experience menopausal symptoms, some for over four years, and currently no optimal treatments exist to provide these women with long-term symptom relief. Many of the most symptomatic women continued to use soy for menopausal symptom relief, even though they were aware of the SEA study results. Given the publications regarding the risks associated with hormonal therapy, greater numbers of menopausal women are seeking alternative therapies, and efforts to identify and test approaches for vasomotor symptom relief are warranted.

\section{Acknowledgment}

This study was funded by the Food and Drug Administration's Office of Women's Health, OMB 0990-0115. The funding agency had no role in the study design or in the collection of the data.

\section{Disclosure}

The authors declare that they have no competing interests in this work. 


\section{References}

1. Kronenberg F. Hot flashes: Epidemiology and physiology. Ann NY Acad Sci. 1990;592:52-86.

2. Daly E, Gray A, Barlow D, McPherson K, Roche M, Vessey M. Measuring the impact of menopausal symptoms on quality of life. $B M J$. 1993;307:836-840.

3. Adlercreutz H, Hamalainen E, Gorbach S, Goldin B. Dietary phytooestrogens and the menopause in Japan. Lancet. 1992;339:1233.

4. Oddens BM. The climacteric cross-culturally: The International Health Foundation South-east Asia Study. Maturitas. 1994;19:155-156.

5. Thom TJ, Epstein FH, Feldman JJ, Leaverton PE. Total morbidity and mortality from heart disease, cancer and stroke from 1950 to 1987 in 27 countries. Bethesda, MD: National Institutes of Health Publication No. 92-3088; 1992.
6. Burke GL, Legault C, Anthony M, et al. Soy protein and isoflavones effects on vasomotor symptoms in peri- and postmenopausal women: The Soy Estrogen Alternative Study. Menopause. 2003;10: $147-153$.

7. Newton KM, Buist DS, Keenan NL, Anderson LA, LaCroix AZ. Use of alternative therapies for menopause symptoms: Results of a populationbased survey. Obstet Gynecol. 2002;100:18-25.

8. Writing Group for the Women's Health Initiative Investigators. Risks and benefits of estrogen plus progestin in healthy postmenopausal women. Principal results from the Women's Health Initiative Randomized Controlled Trial. JAMA. 2002;288:321-333.

\section{Publish your work in this journal}

The International Journal of Women's Health is an international, peerreviewed open-access journal publishing original research, reports, reviews and commentaries on all aspects of women's healthcare including gynecology, obstetrics, and breast cancer. Subject areas include: Chronic conditions (migraine headaches, arthritis, osteoporosis);
Endocrine and autoimmune syndromes; Sexual and reproductive health; Psychological and psychosocial conditions. The manuscript management system is completely online and includes a very quick and fair peer-review system. Visit http://www.dovepress.com/ testimonials.php to read real quotes from published authors. 